\title{
Determination of probe volume dimensions in coherent
}

\section{measurement techniques}

\author{
Sarah A. Tedder, ${ }^{1, *}$ Markus C. Weikl, ${ }^{2}$ Thomas Seeger ${ }^{2}$ and Alfred Leipertz ${ }^{2}$ \\ ${ }^{1}$ Advanced Sensing and Optical Measurement Branch, NASA Langley Research Center, \\ 12 Langley Boulevard, VA, Hampton, USA \\ ${ }^{2}$ Lehrstuhl für Technische Thermodynamik, Am Weichselgarten 8, 91058 Erlangen
} and Erlangen Graduate School in Advanced Optical Technologies (SAOT), Paul-Gordan-Str. 6, 91052 Erlangen, Germany

*Corresponding author: sarah.a.tedder@nasa.gov

When investigating combustion phenomena with pump-probe techniques, the spatial resolution is given by the overlapping region of the laser beams and thus defines the probe volume size. The size of this probe volume becomes important when the length scales of interest are on the same order or smaller. In this work, we present a new approach to measure the probe volume in three dimensions (3-D), which can be used to determine the probe volume length, diameter, and shape. The optical arrangement and data evaluation are demonstrated for a dual-pump dual-broadband coherent anti-Stokes Raman scattering (CARS) setup which is used for combustion diagnostics. This new approach offers a simple, quick alternative with more capabilities than formerly used probe volume measurement methods. $\quad 2008$ Optical Society of America

OCIS codes: 300.6230, 190.4380, 120.1740. 


\section{Introduction}

Coherent nonlinear laser based measurement techniques such as coherent anti-Stokes Raman scattering (CARS) [1-3], laser-induced gratings (LIGS) [4, 5], degenerate four wave mixing (DFWM) [6, 7], polarization spectroscopy (PS) [6, 8] and others usually generate their signal in a region defined by the overlapping region of two or more laser beams. The beam overlap, commonly referred to as the probe volume, is an elongated ellipsoid and in theoretical calculations is normally approximated by a cylinder of length $\mathrm{L}$ and diameter $\mathrm{d}$. The size of this probe volume defines the spatial resolution of the measurement technique and becomes important when the length scales of interest are on the same order or smaller, e.g. in turbulent combustion. Most of the following descriptions are derived from the CARS technique but are mostly also true for other nonlinear techniques.

There exist several methods to measure the probe volume including:

a) Traversing a glass slide with thickness $\mathrm{t}<\mathrm{L}$ and monitoring the level of the non-resonant signal generated in the glass plate in order to measure the probe volume length $[9,10]$.

b) Moving a knife-edge at different positions along the focus and monitoring the intensity of the transmitted laser beams.

c) Traversing a thin gas jet along the probe volume [1].

Each of these methods has limitations. For the first two listed methods the probe volume determination cannot be performed at full laser power used during measurements. This is due to 
destruction of the object, i.e. glass plate or knife edge, which is moved along the focused beams. Hence, either lower laser power needs to be used or a part of the beams has to be sampled which, for method a) involves guiding the generated signal along a different path to the detection system. For the third listed method the thin gas jet is practically hard to achieve, especially when matching the size of small probe volumes on the order of $1 \mathrm{~mm}$. The most often used method is a) which is not applicable in stray-light sensitive techniques like LIGS, PS, DFWM or pure rotational CARS.

In this work we present a new method to determine the probe volume dimensions. This method has more capabilities than the previous mentioned methods, including probe volume determination at full measurement laser power and three-dimensional (3-D) modeling of the probe volume. Additionally this method is useful for combined measurement techniques where the overlap of the two probe volumes has to be verified. A disadvantage of this new method is that an online probe volume determination during the CARS measurements is not possible, because the beam sampler slightly changes the positions of the transmitted beams and therefore the overlap. The beams could also be focused and sampled after the collimating lens allowing for online measurement. However, the aim of accurate probe volume dimension measurement is hindered by a variation of the beam positions due to the additional optics and beam steering from the measured combustion gases.

In order to demonstrate all the capabilities of the new method it was applied to a combined dualpump vibrational and dual-broadband pure rotational CARS system. Based on this example we will describe the optical setup, the data acquisition and the evaluation procedure. Finally, we will present the resulting 3-D model of the probe volume and probe volume length measurements. 
One of these probe volume length measurements will be compared to a glass slide measurement, to assess the accuracy of this new method.

\section{Optical Setup, Phase-Matching, and Data Collection}

A visualization of the radial intensity distribution of the beam overlap region using a CCD camera has been demonstrated by Doerk et al [11] and recently a beam viewing system for the alignment and real-time monitoring of a simultaneous CARS-Rayleigh experiment was presented [12]. These approaches were used as a base for the optical setup of the probe volume measurement method. This setup differs from the beam viewing system with respect to the beam sampling location. Since we do not use the system for real-time monitoring, we only sample the beams during the time the probe volume measurement is conducted.

The optical setup is shown in Fig. 1. A beam sampler, placed directly after the focusing lens, directs $\sim 7 \%$ of the energy of the laser beams to a common intersection point. The beam sampler has no coating on the front surface, is wedged at an angle of 30 arcmin to eliminate internal interference fringes, and is anti-reflective coated on the backside to reduce ghosting. Furthermore, its front surface is specified with a flatness of $\lambda / 10$ to minimize changes to the wave front characteristics. To further reduce the intensity of the light, the beams collected with the beam sampler are passed through neutral density filters which are selected to avoid saturation on the camera. Next, in the optical setup near the focus, the beams are passed through an achromatic 4X-microscope objective which magnifies the beams on a CCD camera which was timed to collect one laser pulse per image. The CCD camera is mounted on a micrometer slide which allows movement along the beam path, indicated in Fig. 1 as the z-direction, so that images can be collected throughout the length of the focus. The images were verified to have minimal changes to wave front characteristics due to the beam sampler and neutral density 
filters. This was done by correspondence of optimum beam overlap in the images with maximum CARS signal with the beam sampler removed.

In order to demonstrate the advantages of this system, it was applied to a dual-pump dualbroadband CARS setup using a folded BOXCARS phases matching geometry shown in Fig. 2. The distance of the beams from the center of the lens was $7 \mathrm{~mm}$ (2xNd_YAG, green, $532 \mathrm{~nm}$ ), $8.85 \mathrm{~mm}$ (broadband dye-laser, red, $683 \mathrm{~nm}$ ) and $7.75 \mathrm{~mm}$ (narrowband dye-laser, orange, $589 \mathrm{~nm}$ ). A focusing lens of $\mathrm{f}=400 \mathrm{~mm}$ is used. In this phase matching arrangement a pure rotational CARS signal (generated by both red and green beams) is collected along with a vibrational CARS signal (generated by the top red, orange, and green beams). An additional CARS signal is also generated in a planar phase matching regime (generated by red aligned with orange and green beams). Although this signal is not collected, its probe volume can be measured for comparison to folded BOXCARS phase matching.

The data are collected, at full measurement power, by taking an image of each beam (while other beams are blocked) at $\mathrm{z}$ locations along the beam path. To illustrate the variation of the laser beams in Fig. 3 images near the focus at $0 \mathrm{~mm}$, and far away at $+10 \mathrm{~mm}$ and $-9 \mathrm{~mm}$ are shown. Normally, only imaging in a region of $4 \mathrm{~mm}$ around the focal point is necessary. Images are collected at equally spaced locations along the $\mathrm{z}$ direction at least $0.25 \mathrm{~mm}$ apart. To ensure that the entire probe volume is measured the collection of data begins at a location where no overlap of the beams is visible and continues in the $\mathrm{z}$ direction until the same condition is met again. These images are then used in the processing steps presented in the next 2 paragraphs.

First the images are preprocessed to identify the beam locations within the images. The images are corrected with regard to background noise and then a threshold of $5 \%$ is applied to the 
individual beam images in order to discriminate the beam location against noise in the images. It was verified that the thresholding at this level has no remarkable influence in the final results. The further evaluation is based on the theoretical CARS equation from reference [13], where $\mathrm{E}_{4}$ is the CARS electric field and $E_{1}, E_{2}, E_{3}$ are the electric fields of the input CARS beams:

$$
E_{4}\left(r_{0}\right)=K^{\prime} e^{-i k_{4} r_{0}} \frac{k_{4}^{2}}{r_{0}} \int E_{1}(\vec{r}) E_{2}^{*}(\vec{r}) E_{3}(\vec{r}) \times e^{i \vec{r} \bullet\left(k_{4} r_{0}+\vec{k}_{2}-\vec{k}_{1}-\vec{k}_{3}\right)} d V
$$

This equation is derived by assuming that the beams are all polarized in the same direction and that the index of refraction of the medium is one. This equation can be further reduced to show that the output field $\mathrm{E}_{4}$ is proportional to the integral of the product of three laser beams' electric fields, as in $E_{4} \propto \int E_{1} E_{2} E_{3} d V$, when assuming that phase matching is satisfied at all beam overlap locations and that the electric fields are coherent. This approximation mirrors the one used in reference [9], which is shown by reference [13] to be a good approximation when only $\mathrm{TEM}_{00}$ modes (Gaussian cross-section profile) are present. Using this approximation, each discrete $(\mathrm{x}, \mathrm{y}, \mathrm{z})$ location within the beam overlap is modeled as an infinitesimal probe volume so that the total CARS electric field is then $E_{4} \propto \sum_{x, y, z} E_{1, x, y, z} E_{2, x, y, z} E_{3, x, y, z}$. In this notation, $\mathrm{E}_{1, \mathrm{x}, \mathrm{y}, \mathrm{z}}$, for example, is the value of the electric field of the first laser at the discrete $(\mathrm{x}, \mathrm{y}, \mathrm{z})$ location. Because an electric field squared is proportional to the intensity, $E^{2} \propto I$, the discrete magnitude of the CARS electric field for each x,y,z location, $E_{4, x, y, z}$, is calculated from the product of the square root of the measured intensities from the images, $\sqrt{I_{1, x, y, z} I_{2, x, y, z} I_{3, x, y, z}}$. After this, to further define the beam overlap, a $1 \%$ threshold is applied to remove any remaining noise. Now, 
a matrix of the discrete magnitudes of the CARS electric field $E_{4, x, y, z} \propto E_{1, x, y, z} E_{2, x, y, z} E_{3, x, y, z}$ at each $\mathrm{x}, \mathrm{y}, \mathrm{z}$ within the beam overlap is available. This information can be used for 3D-modelling.

\section{Results}

A 3-D model of a CARS probe volume including the discrete magnitudes of the CARS electric field is shown in Fig 4. The surfaces of the model are shown transparent with iso-surfaces of $40 \%, 60 \%$ and $90 \%$ of the maximum value of generated CARS-signal. The surfaces are darker towards the center of the volume indicating a higher magnitude for the CARS signal. The 3-D model shows that the shape of the probe volume is an elongated ellipsoid and supports the approximation of a cylinder made by other assessments of the probe volume [1].

To obtain a value for the probe volume length, which is comparable to the glass slide method, the analysis is taken one step further. The discrete magnitudes of the CARS electric field are summed at all $\mathrm{x}$ and $\mathrm{y}$ locations in each $\mathrm{z}$ plane to generate an accumulated magnitude per plane, $E_{4, z} \propto E_{1, z} E_{2, z} E_{3, z}$. A summation of these accumulated magnitudes over all z planes produces a sum-curve, $E_{4}(z)$. This curve is then squared to find the CARS signal intensity sum-curve, shown in Fig. 5. Finally, the 5\% and 95\%-values of the maximum integrated intensity are applied to the sum-curve to determine the length of the probe volume.

The optical setup presented in this work allows spatial resolution comparisons of different CARS phase-matching geometries. The results are shown in Fig. 6 and demonstrate the probe volume length measurement ability of this new approach. Two folded BOXCARS probe volumes, rovibrational and pure rotational, from dual-pump dual-broadband CARS are compared to a planar phase matching scheme of dual-pump CARS. In the planar phase matching case, the length of the probe volume is $47 \%$ larger than the folded BOXCARS vibrational probe volume 
( $\mathrm{L}=1.72 \mathrm{~mm}$ ), similar to predictions of theory and measurements with other probe volume length measurement techniques $[3,10]$. For further comparison, the folded BOXCARS pure rotational probe volume ( $\mathrm{L}=1.80 \mathrm{~mm}$ ) is also plotted. This small difference in the two folded BOXCARS probe volumes can be attributed to the combination of different laser profiles used in the respective cases.

The results achieved for the folded BOXCARS geometry were compared to a measurement using the glass slide technique. The laser beams were attenuated using beam attenuators. A glass slide with thickness $0.1 \mathrm{~mm}$ was traversed through the focus while monitoring the level of nonresonant signal. Due to high scattering from the Nd:YAG laser beam, this measurement could not be performed for pure rotational CARS. The glass slide technique obtained a length of about $1.6 \mathrm{~mm}$ for the vibrational CARS probe volume which is similar to the $1.72 \mathrm{~mm}$ measurements made with the new approach.

Another possible application of this method is the verification of the overlap of two probe volumes being measured simultaneously as is the case with dual-pump dual-broadband CARS. In this CARS phase matching, shown in Fig. 2, a rotational CARS volume and vibrational CARS volume are measured simultaneously using two combinations of three of the four laser beams available. In Fig. 7 the two CARS probe volumes, rotational CARS in red and the vibrational CARS in green indicating that the two CARS signals are measuring the same volume of gas.

\section{Summary}

In summary, a new less-intrusive approach to measure probe volume dimensions in nonlinear optics experiments was presented. This new approach has several advantages above conventional techniques. From the data acquired with this method, it is possible to model the 3-D probe 
volume, to verify coincidence of multiple probe volumes, and to measure probe volume length, diameter and shape. Additionally, problems due to scattering from a narrowband laser in glass slide measurements are not an issue. Hence, the approach is also very useful for stray-light sensitive methods like rotational CARS. Furthermore, the technique allows measurements to be taken at the same laser power as the measurements for the experiment and assist in aligning the signal by optimizing beam overlap. The time needed for this measurement was comparable if not quicker than with other methods. The images used to demonstrate this technique's abilities were taken in less than 15 minutes. This collection time can be of course be significantly reduced by automating the process. From the same set of images, the rotational and vibrational probe volume overlap was verified along with interpretation of spatial resolution. The presented approach is a quick, simple and precise probe volume measurement method. Finally, this experimental approach can be used for other pump-probe techniques, not only for CARS.

\section{Acknowledgements}

The authors gratefully acknowledge funding of the Erlangen Graduate School in Advanced Optical Technologies (SAOT) by the German National Science Foundation (DFG) in the framework of the excellence initiative. The authors would also like to thank Greg Herring and Dimitrii Kozlov for advice and direction regarding CARS probe volume theory.

\section{References}

1. A. C. Eckbreth, Laser Diagnostics for Combustion Temperature and Species (Gordon \& Breach, Amsterdam, Nederland, 1996). 
2. S. O'Byrne, P. M. Danehy, A. D. Cutler, and S. A. Tedder, "Dual-Pump Coherent AntiStokes Raman Scattering Measurements in a Supersonic Combustor " AIAA J. 45, 922-933 (2007).

3. M. C. Weikl, T. Seeger, R. Hierold, and A. Leipertz, "Dual-pump CARS measurements of $\mathrm{N}_{2}, \mathrm{H}_{2}$ and $\mathrm{CO}$ in a partially premixed flame," J. Raman Spectrosc. 38, 983-988 (2007).

4. H.-J. Eichler, P. Günter, and D. W. Pohl, Laser-Induced Dynamic Gratings (SpringerVerlag, Berlin, 1986).

5. T. Seeger, J. Kiefer, M. C. Weikl, A. Leipertz, and D. N. Kozlov, "Time-resolved measurement of the local equivalence ratio in a gaseous propane injection process using laserinduced gratings," Opt. Express 14, 12994-13000 (2006).

6. T. Dreier, and P. Ewart, "Coherent Techniques for Measurements with Intermediate Concentrations," in Applied Combustion Research, K. Kohse-Höinghaus, and J. B. Jeffries, eds. (Taylor and Francis, New York, 2002).

7. T. Dreier, and D. J. Rakestraw, "Measurement of $\mathrm{OH}$ rotational temperatures in a flame using degenerate four-wave mixing," Opt. Lett. 15, 72-74 (1990).

8. J. Kiefer, Z. S. Li, J. Zetterberg, M. Linvin, and M. Aldén, "Simultaneous laser-induced fluorescence and sub-Doppler polarization spectroscopy of the $\mathrm{CH}$ radical," Opt. Commun. 270, 347-352 (2007).

9. D. A. Greenhalgh, "Comments on the use of BOXCARS for gas-phase CARS spectroscopy," J. Raman Spectrosc. 14, 150-153 (1983).

10. T. Seeger, and A. Leipertz, "Experimental comparison of single-shot broadband vibrational and dual-broadband pure rotational coherent anti-Stokes Raman scattering in hot air," Appl. Opt. 35, 2665-2671 (1996). 
11. T. Doerk, J. Ehlbeck, P. Jauernik, J. Stancot, J. Uhlenbusch, and T. Wottka, "Diagnostics of a microwave CO2 laser discharge by means of narrow-band BOXCARS," J. Phys. D 26, 1015-1022 (1993).

12. D. Bivolaru, and G. C. Herring, "Focal-plane imaging of crossed beams in nonlinear optics experiments," Rev. Sci. Inst. 78, 056102 (2007).

13. R. E. Teets, "CARS signals: phase matching, transverse modes, and optical damage effects," Appl. Opt. 25, 855-862 (1986). 


\section{List of figure captions}

Figure 1: (color online)

Experimental setup of probe volume measurement technique.

Figure 2: (color online)

Illustration of the dual-pump dual-broadband folded BOXCARS phase matching scheme used to demonstrate the new technique for probe volume measurements.

Figure 3: (color online)

For each individual beam images at z-locations along the focus spaced by $0.25 \mathrm{~mm}$ are taken. For better visibility only images of z-locations $-9 \mathrm{~mm} / 0 \mathrm{~mm} /+10 \mathrm{~mm}$ are shown in this figure.

Figure 4: (color online)

Representation of the 3-D model of the dual-pump CARS probe volume, $\left(E_{1} E_{2} E_{3}\right)$ as a function of $\mathrm{x}, \mathrm{y}, \mathrm{z}$ or $E_{4, x, y, z}$.

Figure 5:

Integrated magnitude plot giving the length of the probe volume defined as 5\%-95\% of the accumulated intensity.

Figure 6: (color online) 
Comparison of probe volume lengths of different phase-matching geometries, planar and folded BOXCARS, from a dual-pump dual-broadband CARS setup.

Figure 7: (color online)

Verification of overlap of the rotational and vibrational dual-pump dual-broadband CARS 3-D

probe volumes. The scale of $\mathrm{x}$ and $\mathrm{y}$ to $\mathrm{z}$ is four to one. 
Figure 1

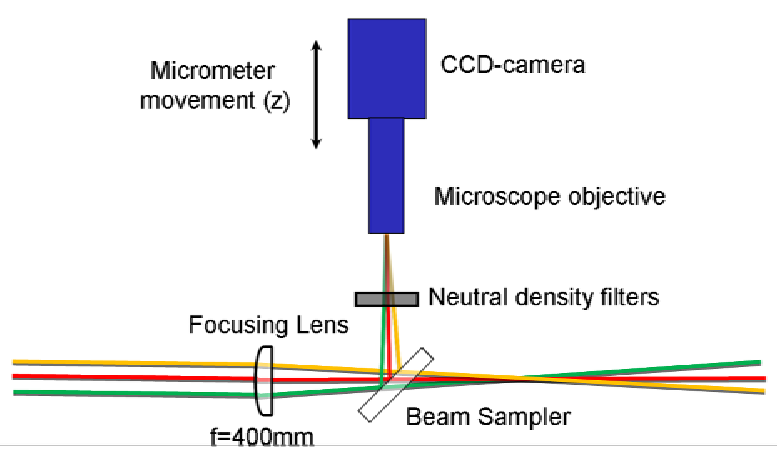




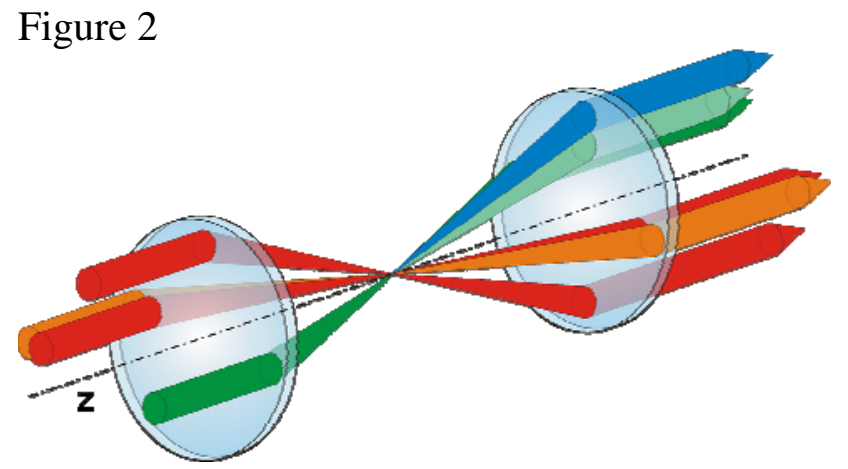


Figure 3

orange

green

$\operatorname{red}_{1}$

$\operatorname{red}_{2}$

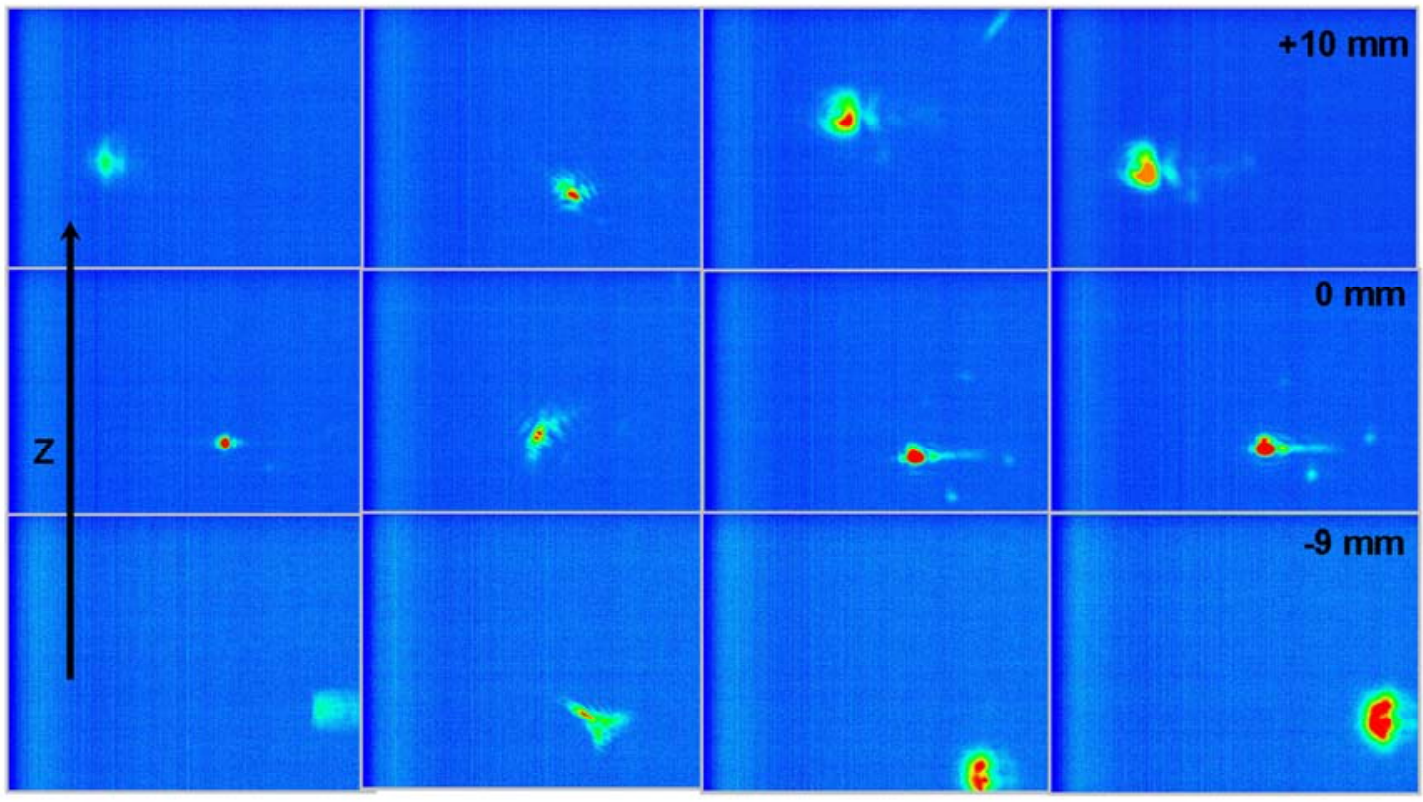


Figure 4

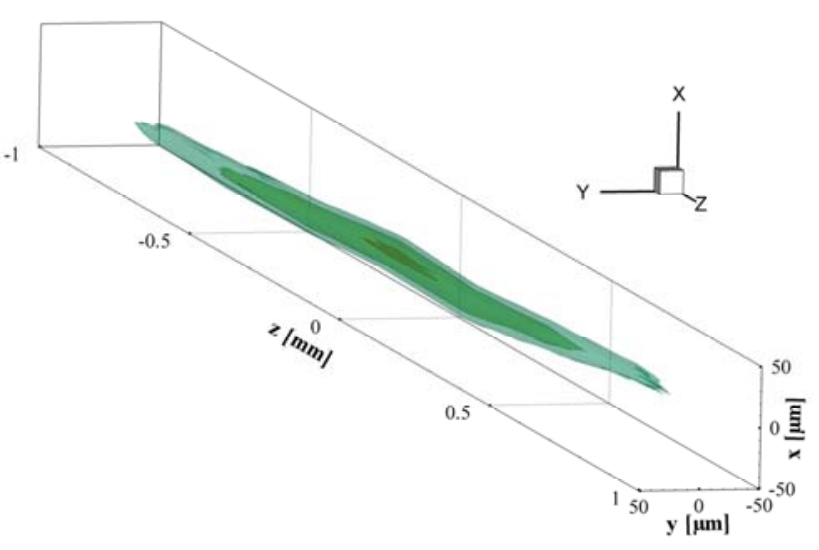


Figure 5

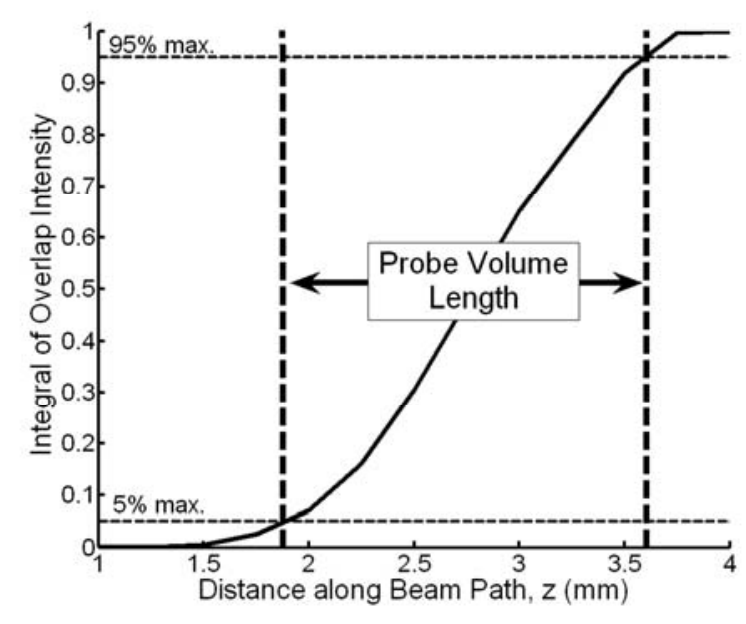


Figure 6

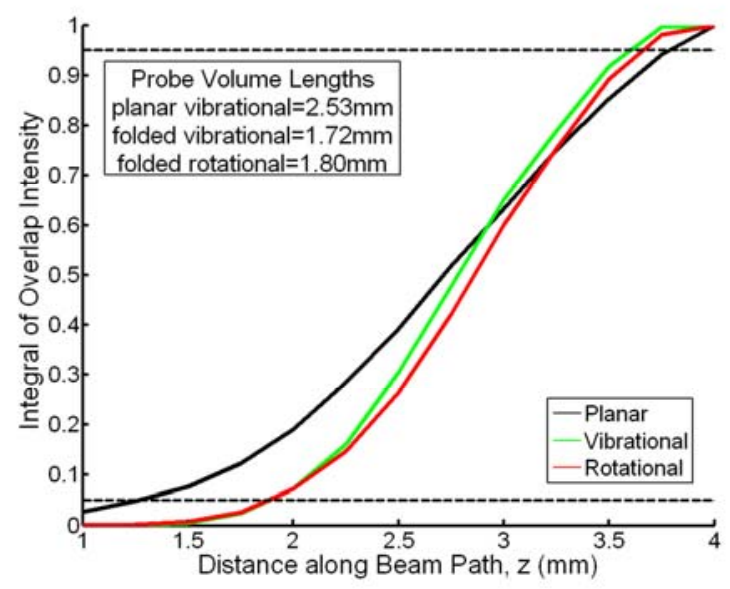




\section{Figure 7

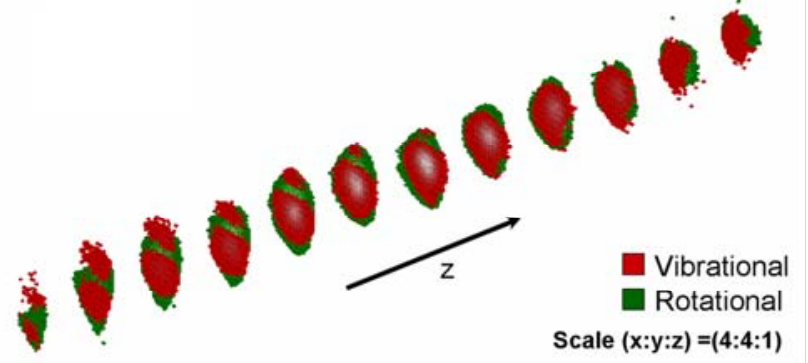

